\title{
Des êtres aux comportements étranges vivent-ils dans les étoiles ${ }^{1}$ ?
}

\section{Do beings with strange behaviours live in the stars?}

\author{
Michel Kreutzer ${ }^{1}$ \\ ${ }^{1}$ Professeur émérite, éthologue, Université Paris Nanterre
}

\begin{abstract}
RÉSUMÉ. Des humains propulsés dans une autre galaxie, posant le pied sur une exo planète propice à certaines formes de vie, sauraient-ils interpréter le comportement des êtres peuplant cet univers extraterrestre ? Sans doute, car en dépit de la grande diversité de formes que pourraient prendre ces créatures, les nécessités de la vie, telles la subsistance et la reproduction, imposent des conduites identifiables. Sous l'infinie variété des comportements possibles, un petit nombre de déterminismes constituent des invariants du vivant.

ABSTRACT. Humans propelled into another galaxy, setting foot on an exoplanet favourable to certain forms of life, could they interpret the behaviour of the beings inhabiting this extra-terrestrial universe? No doubt, because in spite of the great diversity of forms that these creatures could take, the necessities of life, such as subsistence and reproduction, impose identifiable behaviours. Under the infinite variety of possible behaviours, a small number of determinisms constitute living invariants.

MOTS-CLÉS. Vie extra terrestre, contraintes sur les formes, comportements compréhensibles, beauté.

KEYWORDS. Extra terrestrial life, constraint on forms, behaviours understandable, beauty.
\end{abstract}

J'avais à peine quinze ans lorsque j'ai vu, peu après sa sortie en 1959, Voyage au centre de la terre, l'œuvre du réalisateur américain Henry Levin adaptée du roman de Jules Verne, dans laquelle les protagonistes rencontrent et affrontent des créatures fabuleuses. Je n'ai alors pas su m'expliquer pourquoi ce film m'avait tant captivé. Avec le recul du temps, il me semble que j'aie été sensible d'une part à l'imaginaire artistique du cinéaste, d'autre part à la logique scientifique qui sous-tend le récit.

Aujourd'hui, c'est un biologiste épris de rationalisme, Georges Chapouthier, qui m'invite non pas à m'enfoncer dans les entrailles de la Terre mais à explorer d'autres planètes que la nôtre, afin de tenter de répondre à cette question : si la vie a pu émerger au cœur des plus lointains espaces, quelles formes a-t-elle pu prendre?

Nous ne sommes plus dans les années 1950 ; les saisissants «effets spéciaux » mis au point par nos technologies permettent désormais aux amateurs de films de science-fiction de se mouvoir allégrement dans l'espace et le temps. Mais si des vaisseaux spatiaux «atterrissent » en des lieux improbables, habités d'êtres aux formes extravagantes, en revanche les motivations et les actes de ces créatures sont en général immédiatement compréhensibles. C'est là ce qui permet au spectateur d'adhérer spontanément à ces fictions.

Cependant, en tant que biologiste et comportementaliste, c'est d'un œil moins candide que je me propose d'examiner si la conduite d'éventuels êtres extraterrestre pourrait nous être compréhensible. Une vie extraterrestre ne devrait pas pouvoir se manifester autre part que sur des corps célestes appartenant à des systèmes dotés d'un astre « solaire » produisant une énergie qui puisse être captée par des organismes. Par ailleurs, je prendrai le parti de considérer cette vie non pas dans des états

\footnotetext{
${ }^{1}$ Ce texte a bénéficié des remarques et corrections d'Annie et Gérard Dressay que je remercie vivement.
} 
premiers et unicellulaires, mais sous une forme déjà complexe, et donc multicellulaire, celle d'« êtres mosaïque », comme Chapouthier les décrit ${ }^{2}$.

La nature de ces créatures potentielles ne manque évidemment pas de poser bien des questions. Se seraient-elles développées à l'instar de la vie terrestre, qui a pris les deux grandes formes que nous lui connaissons, végétale et animale, ou tout autrement?

Les végétaux sont autotrophes : pour se conserver, ils transforment des ressources minérales en matières organiques à la faveur de l'énergie solaire. Il est notable que l'obligation incontournable d'accomplir ces fonctions vitales n'a pas constitué un obstacle à leur adaptation dans l'eau, le sol et l'air, au point qu'ils ont pu créer en divers milieux une profusion d'espèces aux configurations variées. Comme l'a souligné Portmann ${ }^{3}$, la nature est si prodigue qu'on observe un écart considérable entre les innombrables variétés sous lesquelles elle se présente et le petit nombre de fonctions utilitaires nécessaires à la vie. Cependant, en dépit de cette diversité, ta plupart des plantes présentent des racines, des tiges, des troncs et des feuilles, ces dernières assurant la photosynthèse-

Les animaux sont quant à eux hétérotrophes : pour subsister, il leur faut consommer des nutriments organiques. Les herbivores se nourrissent de plantes, et les carnivores d'animaux qui, pour beaucoup, ne consomment que des plantes. Une multitude de comportements ont été élaborés par les herbivores pour échapper à leurs prédateurs et par les carnivores pour repérer leurs proies. Par exemple, pour se soustraire au regard des prédateurs, les proies se cachent, ont des couleurs cryptiques, ou encore ont développé des aptitudes à la fuite et des systèmes perceptifs leur permettant d'appréhender les dangers et de s'apeurer. De leur côté, les prédateurs doivent chasser seuls ou en groupe ; ils coordonnent leurs activités selon des rythmes adaptés à celles, diurnes ou nocturnes, de leurs proies. On mesure ici combien le besoin d'énergie et de matière organique a suscité de conduites afin que les êtres vivants pourvoient à leur subsistance.

Alors que la majorité des plantes peuvent vivre en étant immobiles, la plupart des animaux doivent leur survie à leur mobilité. La vie végétale étant un préalable à la vie animale, il est loisible d'imaginer que, sur une autre planète, le vivant se contente de cet état végétatif, immobile et paisible. Mais on peut tout autant concevoir l'existence de plantes mobiles comme les virevoltants de nos écosystèmes arides, ou d'autres s'enracinant et se déracinant çà et là, recherchant les meilleurs endroits pour s'exposer à leur «soleil », aux sources d'énergie, et pour puiser des minéraux là où ils sont en nombre. Et puisqu'il 'il existe des animaux consommant des plantes, pourquoi ne pas envisager également des plantes se régénérant de manière traditionnelle mais consommant des animaux comme le font nos plantes carnivores ? Il s'agit là bien sûr d'extrapolations, et d'étendre à un grand nombre de rares exemples observés sur la Terre. Mais serait-il possible de supposer des comportements réellement inédits, dès lors que l'on imagine que les sources de vie sont limitées et engendrent des effets similaires?

Outre la subsistance, on relève bien d'autres fonctions à l'origine des comportements. Sur notre planète, par exemple, tous les êtres, en dépit de leurs efforts pour se conserver, dépérissent et meurent. Mais la reproduction leur permet d'engendrer de nouveaux individus, qui à leur tour poursuivent le cycle de la vie. Sauf à fantasmer que des organismes d'une autre planète aient réalisé le vieux rêve des humains d'une existence éternelle, la vie devrait nécessairement avoir mis en place un système de reproduction analogue à ce qu'il est ici-bas. La reproduction peut être assurée sur Terre de plusieurs manières, dont la plus spectaculaire est sans doute la reproduction sexuée. Chez les plantes, elle est parfois assurée par la production de fleurs attirant les insectes

${ }^{2}$ Georges Chapouthier, L’homme, ce singe en mosä̈que. Editions Odile Jacob, 2001.

${ }^{3}$ Adolf Portmann, La Forme animale, Paris, Editions La Bibliothèque, 2013. 
pollinisateurs et aussi de fruits consommés par les oiseaux, qui en dispersent les graines dans leurs déjections.

La reproduction sexuée est une grande pourvoyeuse de comportements utiles et agréables. Chez les animaux, elle se manifeste par des parades, parfois très complexes, qui leur permettent de s'apparier. La concurrence est souvent grande entre individus pour s'attitrer un partenaire, et la sophistication des sons qu'ils émettent, des couleurs qu'ils déploient et des odeurs qu'ils sécrètent, tout à fait saisissante. Pourquoi dépenser autant d'énergie pour simplement mettre des gamètes en contact? Darwin ${ }^{4}$ voyait dans le choix des femelles la manifestation d'un goût pour le beau. Mais comme ces couleurs et ces sons produits par les mâles à des fins de séduction nous apparaissent « beaux » à nous aussi, humains, quoiqu'ils ne nous soient pas destinés, ils sont la preuve, selon cet auteur, que la beauté est conçue de la même manière chez des espèces différentes. J'ai par ailleurs soutenu que les animaux sont, comme nous, des êtres hédonistes, pour qui vivre est indissociable d'une motivation à rechercher des plaisirs et à éviter des déplaisirs ${ }^{5}$.

Quantité d'espèces assurent la survie de leurs jeunes en les protégeant et en leur assurant des soins, notamment quand ils naissent immatures. Ces comportements parentaux occupent bien des individus de nombreuses espèces, et chez les descendants des dinosaures que sont les oiseaux, outre la construction du nid et la couvaison, ils atteignent des sommets de complexité. Mais il existe des cas où les jeunes naissent totalement autonomes, et sont capables d'assurer immédiatement leur subsistance. La diversité des comportements est là aussi immense.

Pour assurer leur survie et leur reproduction, les animaux se trouvent à la fois en compétition et en coopération. L'usage qu'ils font de l'espace et du temps les amène à vivre tantôt séparément et tantôt en groupe ; ils sont tantôt sédentaires et tantôt en migration. La compétition pour exister et se reproduire, pour bien vivre, les conduit à défendre âprement des territoires ou bien des statuts hiérarchiques avantageux. Si, comme nous le supposons, les mêmes besoins sont sur d'autres planètes indispensables au vivant, alors des nécessités identiques ont entraîné des solutions comparables. Comparables mais pas forcément similaires. Il y a cent millions d'années, la vie sur Terre existait déjà, mais les espèces étaient bien différentes des espèces actuelles, quoiqu'elles dussent assurer leur survie et leur reproduction. Et nous postulons que ce ne sont pas des machines mais des êtres sensibles qui percevaient et ressentaient ces formes d'existence.

Les voyageurs de Jules Verne explorant le centre de la Terre reconnaîtraient facilement ce qui anime les créatures de nos actuels films de science-fiction, mais identifieraient-ils aussi facilement les conduites d'êtres vivant sur de lointaines planètes ? Je répondrai sans conteste par l'affirmative, du simple fait que toute créature doit mener une vie de relation et élaborer des comportements pour faire face à la nécessité de subsister et de se reproduire. Quant à la question portant sur la beauté ou la laideur des extraterrestres on trouve chez Spinoza ${ }^{6}$ de quoi en tempérer la pertinence : « $L a$ beauté, très illustre ami, n'est pas tant une qualité de l'objet considéré qu'un effet en celui qui le regarde. »

\footnotetext{
${ }^{4}$ Charles Darwin, The Descent of Man and Selection in Relation to Sex, Londres, John Murray, 1871.
${ }^{5}$ Michel Kreutzer, «Extension du concept d'hédonisme. Repenser les désirs et satisfactions des animaux ». In L'animal désanthropisé, dir. Eric Baratay, Édition de la Sorbonne, 2021, p.119-131.

${ }^{6}$ Benedict de Spinoza, Lettre à Hugo Boxel, 1674, Correspondance, Euvres complètes, Bibliothèque de la Pléiade, Gallimard, 1967, p. 1238.
} 


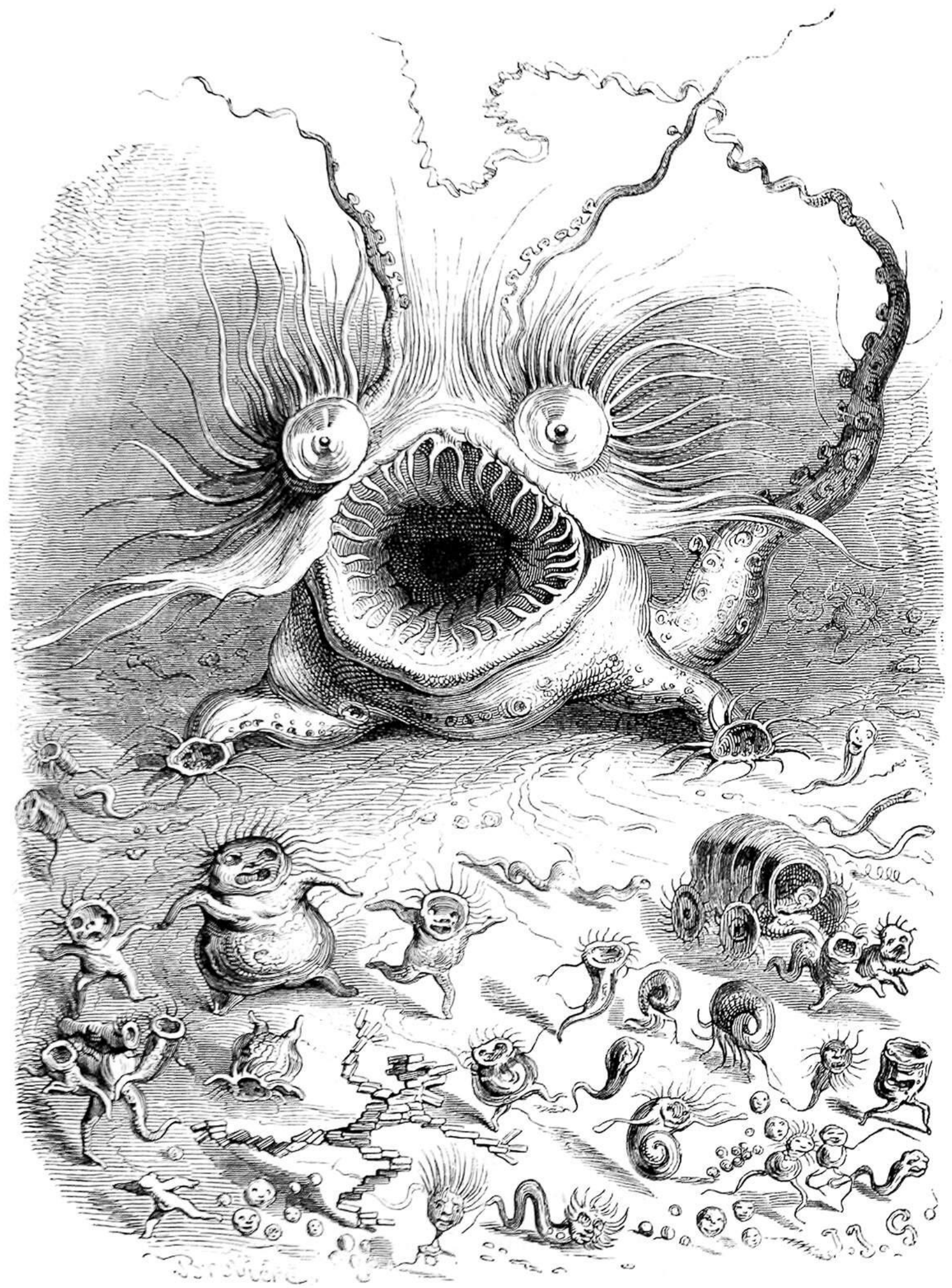

Le Volvoce, dessin de Jean-Jacques Grandville (1803-1847) extrait de : Un autre monde, texte de Taxile Delord, ouvrage édité à Paris en 1844 par Henri Fournier. 